\title{
Pulse transit time as a measure of respiratory effort under noninvasive ventilation
}

\author{
Olivier Contal*,f, Claudio Carnevale ${ }^{\#, f}$, Jean-Christian Borel ${ }^{\uparrow,+, \S,}$ \\ AbdelKébir Sabil ${ }^{\uparrow,+, \S}$, Renaud Tamisier ${ }^{\uparrow,+, \S}$, Patrick Lévy ${ }^{\top,+, \S}$, \\ Jean-Paul Janssens ${ }^{\star, * *}$ and Jean-Louis Pépin ${ }^{\top,+, \varsigma_{, * *}}$
}

ABSTRACT: Among the respiratory events that may occur during nocturnal noninvasive ventilation (NIV), differentiating between central and obstructive events requires appropriate indicators of respiratory effort. The aim of the present study was to assess pulse transit time (PTT) as an indicator of respiratory effort under NIV in comparison with oesophageal pressure (Poes).

During wakefulness, PTT was compared to Poes during spontaneous breathing and under NIV with or without induced leaks in 11 healthy individuals. In addition, the contribution of PTT versus Poes to differentiation of central from obstructive respiratory events occurring under NIV during sleep was evaluated in 10 patients with obesity hypoventilation syndrome (OHS).

From spontaneous breathing to NIV without leaks, respiratory effort decreased significantly whereas, with increasing level of leaks, there was a significant increase in respiratory effort. Changes in PTT accurately reflected changes in Poes. In OHS patients during nocturnal NIV, intraclass correlation coefficients between Poes and PTT were 0.970 for total number of events and 0.970 for percentage of central events.

PTT accurately reflects the unloading of respiratory muscles induced by NIV and the increase in respiratory effort during leaks. PTT during sleep is also useful to differentiate central from obstructive respiratory events occurring under NIV.

\section{KEYWORDS: Monitoring, noninvasive ventilation, sleep medicine}

$\mathbf{N}$ oninvasive ventilation (NIV) is widely accepted as a long-term treatment for chronic hypercapnic respiratory failure. Monitoring of the efficacy of long-term NIV usually relies on medical history, daytime arterial blood gases and nocturnal pulse oximetry, sometimes coupled with transcutaneous capnography [1]. Recently, an increasing awareness of nocturnal respiratory events occurring under NIV has led to a wider use of respiratory polygraphy and polysomnography (PSG) to improve adjustment of long-term NIV settings [2-5]. The most frequent problems detected are unintentional leaks (i.e. not related to exhalation valve of interface), patientventilatory asynchrony, and obstructive or central events (either residual or induced by NIV) [4].

Differentiating between central and obstructive events on polygraphy or PSG tracings under NIV is more challenging than in spontaneous breathing and requires appropriate measurements of respiratory effort. Oesophageal pressure $\left(P_{\text {oes }}\right)$ is the "gold standard" for quantifying variations in intrathoracic pressure resulting from respiratory efforts. However, measuring $P_{\text {oes }}$ is invasive, frequently unaccepted or poorly tolerated by the patient and interferes with sleep quality. Pulse transit time (PTT) has been proposed as an alternate means for quantifying respiratory effort by detecting changes in blood pressure oscillations associated with pleural pressure swings [6]. PTT refers to the time it takes for a pulse wave to travel between two arterial sites. The speed at which this arterial pressure wave travels is inversely proportional to blood pressure. The variation of PTT between inspiration and expiration reflects the intensity of corresponding changes in intrathoracic pressure. Our group has previously demonstrated that PTT can be used during PSG to analyse respiratory effort and, moreover, that it is specific when defining certain respiratory events occurring during spontaneous breathing (hypopnoeas, upper airway resistance episodes and central events) [7-9]. However, to date, no study has addressed the interest of PTT for characterising nocturnal respiratory events occurring under
AFFILIATIONS

${ }^{*}$ Division of Pulmonary Diseases, Geneva University Hospitals, Geneva, Switzerland.

\#Dipartimento Toraco-Polmonare e Cardio-Circolatorio, University of Milan, IRCCS Fondazione Cà Granda, Ospedale Maggiore Policlinico, Milan, Italy.

"Grenoble University Hospital, Pole de Rééducation et Physiologie, +INSERM U1042, HP2 Laboratory (Hypoxia, Pathophysiology), and ${ }^{\S}$ Grenoble University Hospital, Sleep Laboratory, EFCR, Grenoble, France. ${ }^{f}$ The two first authors contributed equally to the work.

**The senior authors contributed equally to the work.

\section{CORRESPONDENCE}

J-L. Pépin

Grenoble University Hospital

Pole de Rééducation et Physiologie Grenoble

F-38043

France

E-mail: JPepin@chu-grenoble.fr

Received:

Nov 072011

Accepted after revision:

April 052012

First published online:

April 202012

European Respiratory Journal Print ISSN 0903-1936

Online ISSN 1399-3003 
NIV. Because NIV modifies intrathoracic pressures, and thus may affect blood pressure oscillations associated with pleural pressure swings, the contribution of PTT measurements in this setting must be evaluated. Also, NIV reduces respiratory effort, which may therefore affect the sensitivity of PTT in detecting changes in inspiratory effort [10].

We undertook the present study to assess the reliability of PTT for: 1) quantifying respiratory effort in comparison with Poes in healthy awake individuals, while breathing spontaneously, then under NIV with or without unintentional leaks; and 2) for differentiating central from obstructive events occurring during sleep under NIV in patients with obesity hypoventilation syndrome (OHS).

\section{MATERIALS AND METHODS}

\section{Daytime assessment of respiratory effort in healthy individuals}

The objective of this experiment was to analyse the contribution of PTT to identifying and quantifying changes in respiratory muscle effort using Poes as the gold standard (fig. 1).

\section{Set-up and measurements}

Healthy subjects were studied while awake and breathing spontaneously or under NIV. Respiratory effort was measured by $P$ oes as reference method and compared to PTT as previously described $[7,8]$. Respiratory flow and mask pressure (pneumotachograph), thoracic and abdominal movements, arterial oxygen saturation, and ECG were monitored by polygraphy (Deltamed $^{\mathrm{TM}}$, San Carlos, CA, USA).

After 5 min of recording during spontaneous breathing, subjects were ventilated consecutively with two different bi-level pressure support devices (GoodKnight 425ST (Tyco Healthcare Inc., Nancy, France) and VPAP III ST (ResMed, North Ryde, Australia)). Two devices were used because we hypothesised that their pressurisation characteristics, and thus their impact on respiratory effort, might differ. The same settings were used in all situations: inspiratory positive airway pressure (IPAP) $15 \mathrm{cmH}_{2} \mathrm{O}$, expiratory positive airway pressure $5 \mathrm{cmH}_{2} \mathrm{O}$, backup rate 8 per minute and inspiratory time $1.8 \mathrm{~s}$. A leak generator was used as described in a previous study [11]. The system included a computer-driven solenoid valve that could be opened from 0 to $10 \mathrm{~mm}$, thus generating a maximal unintentional leak of $60 \mathrm{~L} \cdot \mathrm{min}^{-1}$ at $15 \mathrm{cmH}_{2} \mathrm{O}$ of IPAP. Four levels of leaks were generated $\left(0,24,40\right.$ and $\left.60 \mathrm{~L} \cdot \mathrm{min}^{-1}\right)$ in a random order for 5 min each.

\section{Digital signal processing and analysis of polygraph recordings}

All the digital signal processing and analysis was performed using dedicated engineering Matlab software (version 6.5.0; The MathWorks Inc., Natick, MA, USA). We first selected, by visual analysis, valid portions of the tracings with at least 10 cycles with no artefacts on any of the analysed signals (flow, PTT and Poes). Limits of each respiratory cycle were automatically defined on the flow signal as previously described by BACHY et al. [12]. The Poes signal classically presents a specific high-frequency noise generated by the heart beats: this could affect quantification of effort when calculating the area under the curve of the negative deflection of the Poes signal. Adaptive filtering using a "Least Mean Square" algorithm was used to solve this problem. The beginning of the respiratory cycle was

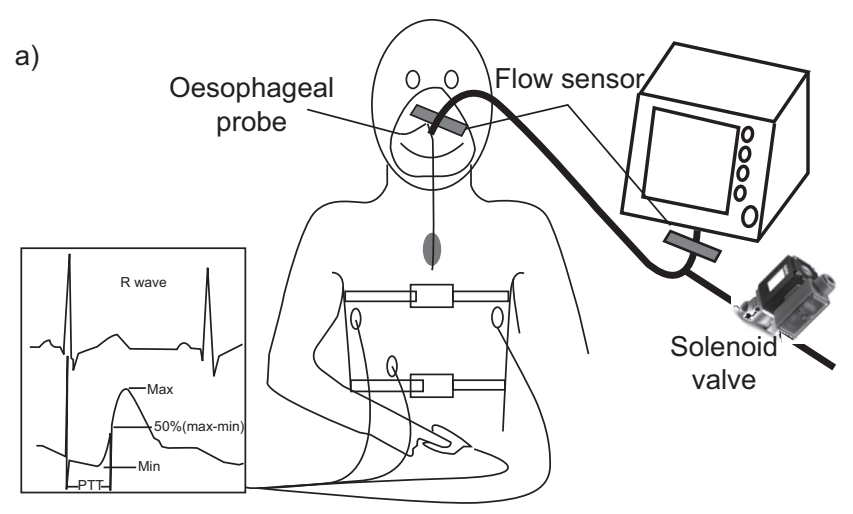

b)

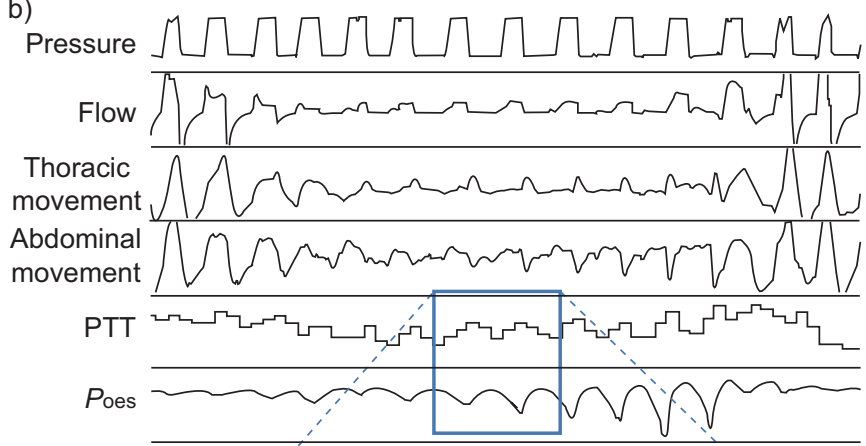

c)
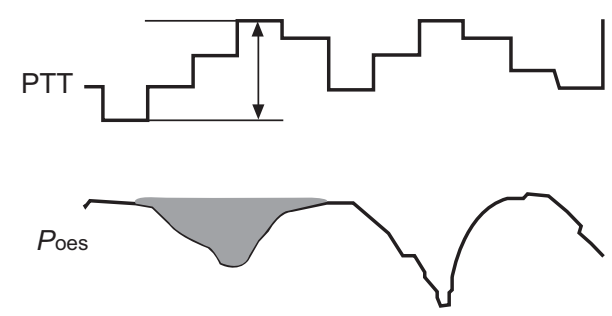

FIGURE 1. Diagram of experiments. a) Healthy subject ventilated and monitored with oesophageal probe, flow sensor, thoracic and abdominal sensors, ECG, and oximetric photoplethysmography (for pulse transit time (PTT)), and a computer-controlled solenoid valve generating calibrated unintentional leaks. b) Tracing during nocturnal experiment in an obesity hypoventilation syndrome patient. c) Enlargement of box in (b) showing PPT (measure of time on one cycle from the expiratory peak to the inspiratory peak) and oesophageal pressure (Poes) (calculation of the area under zero). Max: maximum; min: minimum.

used to readjust the zero level for each cycle on the Poes signal. This was necessary to correct for the intermittent drift that occurred for the Poes signal. The area under the curve of the inspiratory Poes signal was then calculated, for each cycle. PTT swings were quantified as previously described [13] by measuring the difference between the expiratory peak and the inspiratory nadir for each cycle (fig. 1c).

\section{Use of PTT as a surrogate marker of inspiratory effort to discriminate between central and obstructive respiratory events during sleep in patients with OHS}

The aim of this experiment was to determine whether it was possible, based on PTT, to determine whether decreases in flow occurring under NIV during sleep were of a central or obstructive nature, using Poes as the gold standard. 


\section{Set-up, definition of events and measurements}

Patients with OHS chronically treated at home with nocturnal bilevel positive pressure ventilation for $\geqslant 3$ months were included. OHS was defined as the association of obesity (body mass index (BMI) $>30 \mathrm{~kg} \cdot \mathrm{m}^{-2}$ ) and daytime hypercapnia without any other obstructive or restrictive pulmonary pathology [14, 15]. Exclusion criteria were: age $<18$ yrs; forced expiratory volume in $1 \mathrm{~s} /$ forced vital capacity ratio $<70 \%$; history of an acute episode of cardiac and/or respiratory failure within the previous 3 months; allergy to latex; and a history of oesophageal varices precluding the use of an oesophageal probe. PSG under NIV was performed as previously described by our team [16, 17] using a single bi-level device (VPAP ST III; ResMed, Syndey, Australia) with the patients' usual ventilator settings (table 1). Respiratory events under NIV were scored manually according to criteria proposed by GONZALEZ-BERMEJO et al. [4].

\section{Data analysis}

Scoring of respiratory events focused on central, mixed and obstructive events. Figure 2 shows representative examples of how events were identified as either central or obstructive (partial or total upper airway obstruction with or without reduction of ventilatory drive) by using PTT and Poes.

First, respiratory events were scored by one observer based on Poes alone while blinded to the PTT signal. Secondly, events were scored using the PTT signal as indicator of respiratory effort, while the same observer was blinded to the Poes trace.

\section{Ethical approval}

All subjects provided written informed consent. Study protocol was approved by the Ethics Committee of Grenoble University

\begin{tabular}{|c|c|c|}
\hline TABLE 1 & \multicolumn{2}{|c|}{$\begin{array}{l}\text { Respiratory function, blood gases (room air) and } \\
\text { noninvasive ventilation (NIV) settings of patients } \\
\text { with obesity hypoventilation syndrome }\end{array}$} \\
\hline \multicolumn{2}{|l|}{ VC L } & $3.2 \pm 1$ \\
\hline \multicolumn{2}{|c|}{ VC \% pred } & $88 \pm 23$ \\
\hline \multicolumn{2}{|c|}{ FEV $_{1} \mathrm{~L}$} & $2.4 \pm 0.8$ \\
\hline \multicolumn{2}{|c|}{ FEV $1 \%$ pred } & $83 \pm 25$ \\
\hline \multicolumn{2}{|c|}{$\mathrm{FEV}_{1} / \mathrm{VC}$} & $79.3 \pm 8.6$ \\
\hline \multicolumn{2}{|c|}{$\mathrm{FEV}_{1 / \mathrm{FVC}} \%$ pred } & $94.3 \pm 1.1$ \\
\hline \multicolumn{3}{|c|}{ Arterial blood gases } \\
\hline \multicolumn{2}{|l|}{$\mathrm{pH}$} & $7.40 \pm 0.03$ \\
\hline \multicolumn{2}{|c|}{$\mathrm{Pa}, \mathrm{O}_{2} \mathrm{kPa}$} & $10 \pm 1.7$ \\
\hline \multicolumn{2}{|c|}{$\mathrm{Pa}, \mathrm{CO}_{2} \mathrm{KPa}$} & $5.9 \pm 0.8$ \\
\hline \multicolumn{2}{|c|}{$\mathrm{HCO}_{3}^{-} \mathrm{mmol} \cdot \mathrm{L}^{-1}$} & $27 \pm 1.7$ \\
\hline \multicolumn{2}{|c|}{$\mathrm{Sa}, \mathrm{O}_{2} \%$} & $95 \pm 1.7$ \\
\hline \multicolumn{3}{|c|}{ NIV settings } \\
\hline \multicolumn{2}{|c|}{ Back-up rate cycles $\cdot \mathrm{min}^{-1}$} & $13 \pm 2$ \\
\hline \multicolumn{2}{|c|}{ IPAP $\mathrm{cmH}_{2} \mathrm{O}$} & $19 \pm 2$ \\
\hline \multicolumn{2}{|c|}{ EPAP $\mathrm{cmH}_{2} \mathrm{O}$} & $11 \pm 2$ \\
\hline
\end{tabular}

Data are presented as mean \pm SD. VC: vital capacity; \% pred: \% predicted; FEV1: forced expiratory volume in $1 \mathrm{~s}$; $\mathrm{FVC}$ : forced vital capacity; $\mathrm{Pa}_{1} \mathrm{O}_{2}$ : arterial oxygen tension; $\mathrm{Pa}_{2} \mathrm{CO}_{2}$ : arterial carbon dioxide tension; $\mathrm{HCO}_{3}$ : bicarbonate; $\mathrm{Sa}, \mathrm{O}_{2}$ : arterial oxygen saturation; IPAP: inspiratory positive airway pressure; EPAP: expiratory positive airway pressure.
Hospital Center (Grenoble, France). This study is registered at www.clinicaltrials.gov with identifier NCT00983411.

\section{Statistical analysis}

Normality of the data was checked using tests of skewness and kurtosis, and equality of variance was assessed by Levene's test. Data are presented as median (interquartile range) when appropriate. PTT and Poes values are reported as percentage of value during spontaneous breathing.

Comparisons between experimental conditions were analysed by two-way repeated-measures ANOVA with a repeated factor "condition" (no leaks, and calibrated leaks of 24, 40 and $60 \mathrm{~L} \cdot \mathrm{min}^{-1}$ versus spontaneous breathing), and a repeated factor "ventilator" (GoodKnight 425ST versus VPAP III). Bonferroni's correction was used for the paired t-tests.

Comparisons between PTT and Poes for the total number of events and percentage of central events occurring under NIV in OHS patients was expressed as proposed by BLAND and Altman [18]. The agreement between PTT and Poes for events occurring during sleep was quantified using an intraclass correlation coefficient (ICC) calculated with SPSS Statistics 17 (SPSS Inc, Chicago, IL, USA) [19]. ICC values between 0.51 and 0.70 describe moderate reproducibility, and values above 0.71 ,

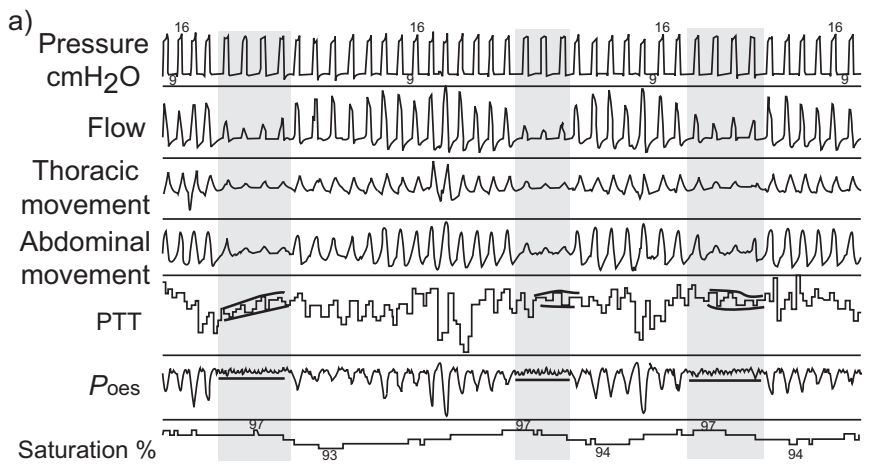

b)

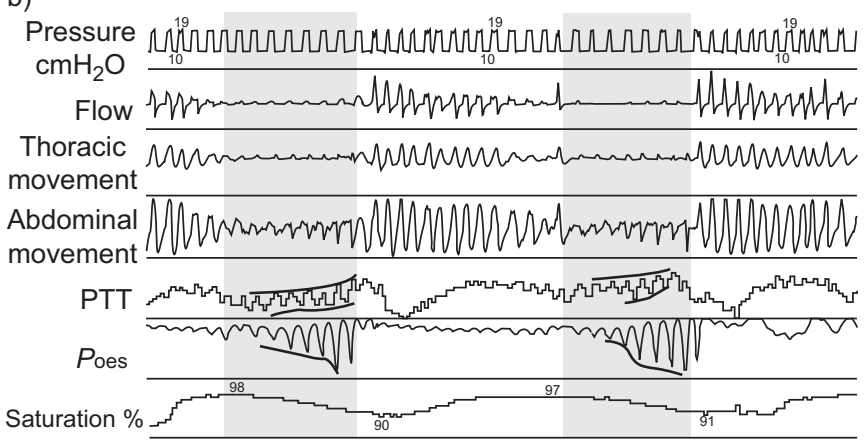

FIGURE 2. Illustrative examples of how visual scoring was performed to identify central versus obstructive events using pulse transit time (PTT) or oesophageal pressure (Poes). A 3-min epoch is shown. a) Central respiratory event: for both Poes and PTT signals, respiratory oscillations are markedly reduced or disappear. b) Obstructive respiratory event: swings of Poes become increasingly negative during an obstructive event, with a simultaneous increase in oscillations of PTT between inspiration and expiration (dark lines). 

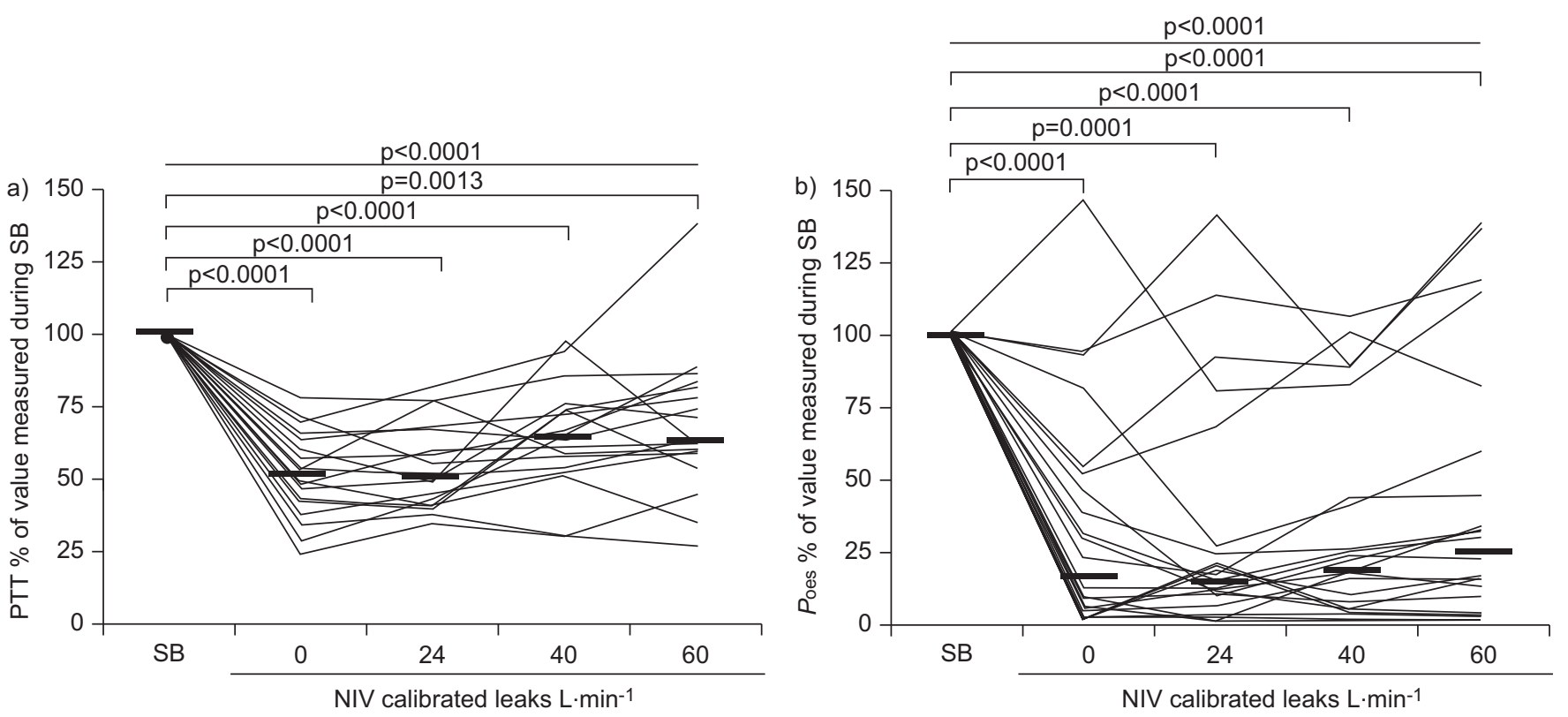

FIGURE 3. Respiratory effort measured by a) pulse transit time (PTT) or b) oesophageal pressure (Poes) in awake healthy subjects during spontaneous breathing (SB) or under noninvasive ventilation (NIV) at different levels of leaks. The changes in Poes and PTT were obvious during NIV without leaks, a situation associated with a complete rest of respiratory muscles. In response to leaks, some normal subjects exhibited a higher percentage of triggered cycles and clearly increased their respiratory effort. This was nicely demonstrated by Poes but less clearly shown by PTT. Horizontal lines represent median values.

good reproducibility. Correlations were assessed by Pearson's or Spearman's coefficients depending upon the normality of distribution.

For all tests, a level of significance of $\mathrm{p}<0.05$ was used. Statistical analysis was performed with NCSS 97 software (NCSS, Kaysville, UT, USA).

\section{RESULTS}

\section{Daytime assessment of respiratory effort in healthy} individuals

11 subjects (six males and five females; age $31 \pm 9$ yrs; BMI $22.3 \pm 2.5 \mathrm{~kg} \cdot \mathrm{m}^{-2}$ ) were included. Respiratory effort was assessed simultaneously by oesophageal probe and PTT. Subjects were recorded: 1) during spontaneous breathing; 2) under NIV without leaks; and 3) under NIV with induced calibrated leaks applied in a random order. The mathematical processing of PTT and $P$ oes is shown in figure 1. A two-way, repeated-measures
ANOVA with a repeated factor "condition" (no leaks, or calibrated leaks of 24,40 and $60 \mathrm{~L} \cdot \mathrm{min}^{-1}$ at inspiratory pressure versus spontaneous breathing) and a repeated factor "ventilator" (GoodKnight 425ST versus VPAP III) showed no effect related to the ventilator. As such, figure 3 and table 2 present mean values for the two ventilators. There was a significant decrease in respiratory effort when shifting from spontaneous breathing to NIV without leaks (fig. 3). This change was highly significant with either PTT or Poes. When leaks were increased (from 0 to $\left.60 \mathrm{~L} \cdot \mathrm{min}^{-1}\right)$, increase in respiratory effort was documented similarly by PTT and Poes (table 2).

\section{Assessment of OHS patients during sleep}

10 patients with OHS (six males and four females; aged $56 \pm 12 \mathrm{yrs}$; BMI $35 \pm 5 \mathrm{~kg} \cdot \mathrm{m}^{-2}$ ) were included in the night-time study. Table 1 summarises respiratory function, blood gases and usual NIV settings of these patients.

TABLE 2 Respiratory effort measured by pulse transit time (PTT) or oesophageal pressure (Poes) in awake normal subjects during spontaneous breathing (SB) or under noninvasive ventilation (NIV) at different levels of leaks

SB

NIV

p-value

\section{No leaks}

\section{Calibrated leak L. $\mathrm{min}^{-1}$}

\section{0}

$\begin{array}{lccccc}\text { PTT } & 100 & 51.3(41.2-64.3) & 50.6(41.3-67.4) & 64.3(53.7-75.0) & 63.1(58.2-82.3) \\ \text { Poes } & 100 & 16.8(3.3-51.8) & 15.1(8.3-36.4) & 19.2(4.4-52.5) & <0.0001\end{array}$

$63.1(58.2-82.3)$ $25.5(7.5-64.3)$
$<0.0001$ $<0.0001$

Data are presented as median (interquartile range), unless otherwise stated. Values are expressed as percentage of SB value. See main text for mathematical processing of Poes and PTT signals. " : two-way, repeated-measures ANOVA. 

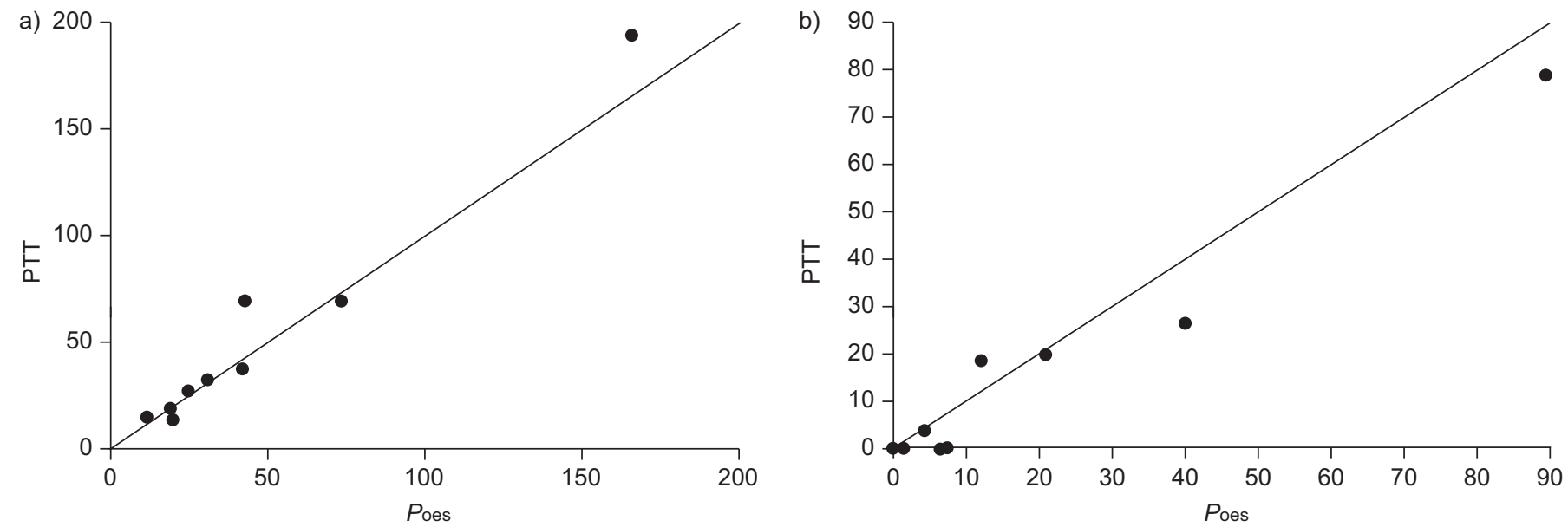

FIGURE 4. Representation of scoring of all respiratory events and percentage of central events $(r=0.98$ and $r=0.86$ respectively, $p=0.001)$ a) Correlation between total number events scored when using pulse transit time (PTT) alone versus oesophageal pressure (Poes) alone. b) Correlation between percentage of central events scored when using PTT alone versus Poes alone.

Based on $P_{\text {oes, }}$ we found that our patients had a mean \pm SD total number of respiratory events on NIV of $43 \pm 48$ per night. These events were predominantly obstructive in nature $(85.8 \%$ of total number of events). The total number of events and the percentage of central events scored when using PTT alone or Poes alone were highly significantly correlated $(\mathrm{r}=0.98$ and $\mathrm{r}=0.86$, respectively; $\mathrm{p}=0.001$ ) (fig. 4 ). On the corresponding Bland-Altman plot, the mean systematic bias was five events (limits of agreement -19.3-29.3 events) and $-3.4 \%$ (limits of agreement -14.9-8.2\%), respectively. The ICCs between Poes and PTT were $0.970(95 \%$ CI $0.889-0.992, \mathrm{p}<0.0001)$ for total number of events and 0.970 (95\% CI 0.871-0.993, p<0.0001) for percentage of central events (table 3 ).

An additional interesting feature of PTT was its ability to describe changes in respiratory effort when respiratory cycles were triggered or not by the patients (fig. 5).

\section{DISCUSSION}

Data presented in the present study show that, in normal subjects, in spite of the changes in intrathoracic pressure and haemodynamics induced by positive pressure ventilation, PTT accurately reflects the unloading of respiratory muscles induced by NIV, as well as the increase in respiratory effort accompanying leaks during NIV. This may be of interest when seeking to unload the respiratory muscles during daytime titration of NIV. We also showed that the analysis of PTT during sleep studies allows an appropriate classification of respiratory events occurring under NIV as being either central or obstructive in nature (i.e. partial or complete upper airway obstruction with or without reduction of ventilator drive) [4]. These results support the use of PTT as a surrogate marker of inspiratory muscle effort under NIV.

Complex respiratory events occurring during sleep under NIV, which may have a detrimental effect on both quality of sleep and control of nocturnal hypoventilation, have been the focus of several recent studies and reviews $[2,20]$. In a PSG study, $40 \%$ of obese patients using NIV showed a high index of periodic breathing, mostly occurring in light sleep and associated with sometimes severe nocturnal hypoxaemia [5]. Identification of these events is important in order to select the appropriate changes in ventilator settings and/or interface. Whether systematic PSG is necessary for titrating NIV, as suggested by the American Academy of Sleep Medicine [2], is a subject of debate: this option is not supported in a recent review on monitoring of home NIV [21]. However, when PSG or polygraphy are performed, determining whether events are primarily central or obstructive is often critical (i.e. with a decrease or increase in respiratory drive). This is especially the

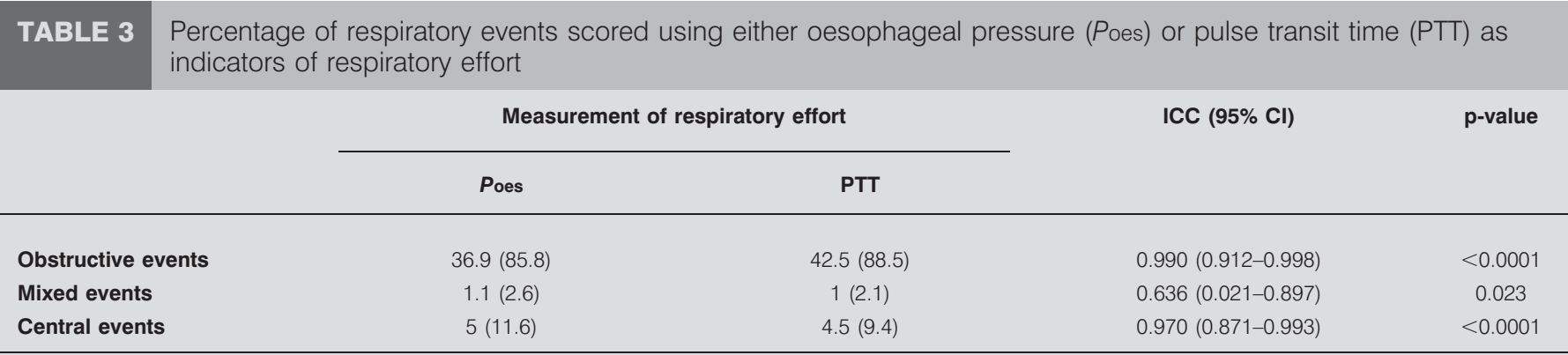

Data are presented as mean number of events (\% of total number of events), unless otherwise stated. ICC: intraclass correlation coefficient. 
a)
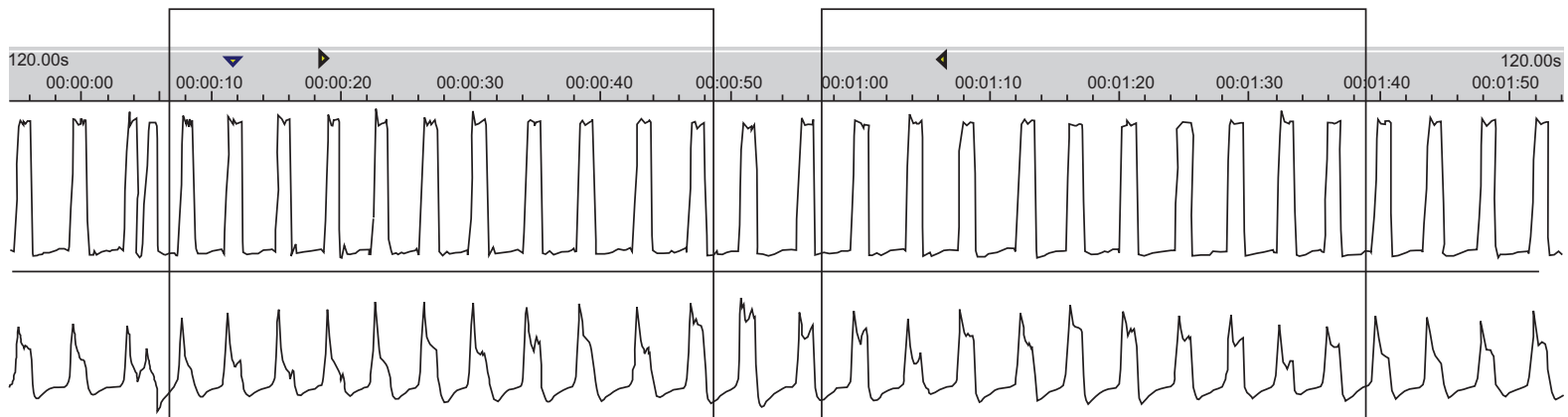

Thoracic

movement

Abdominal

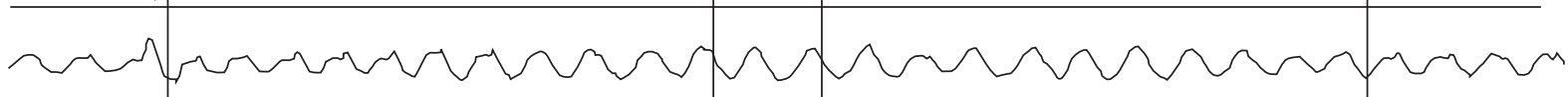

movement

PTT
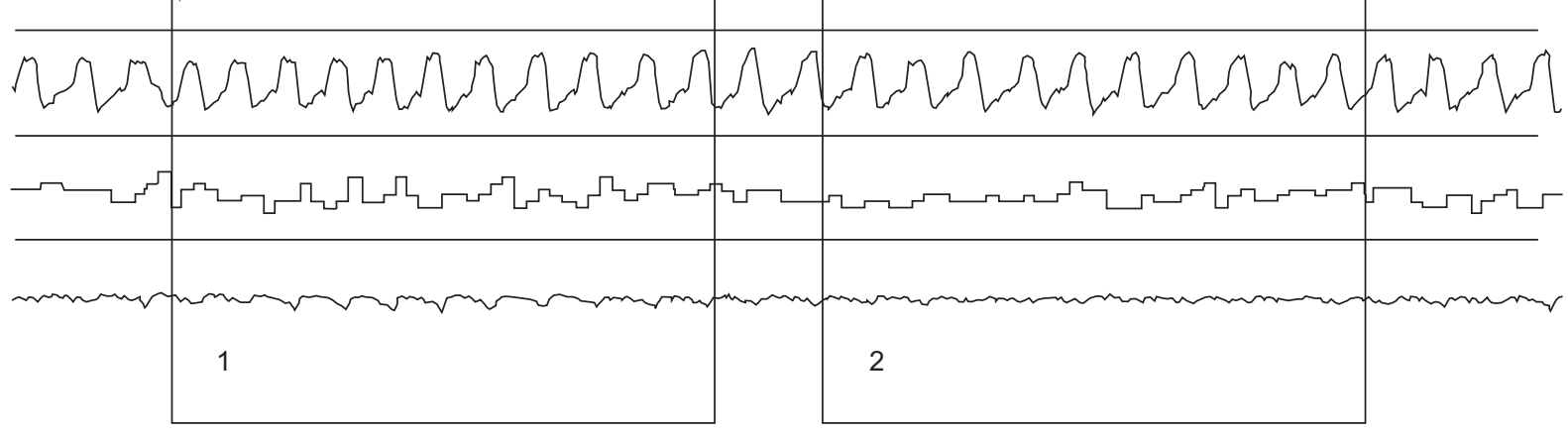

b)

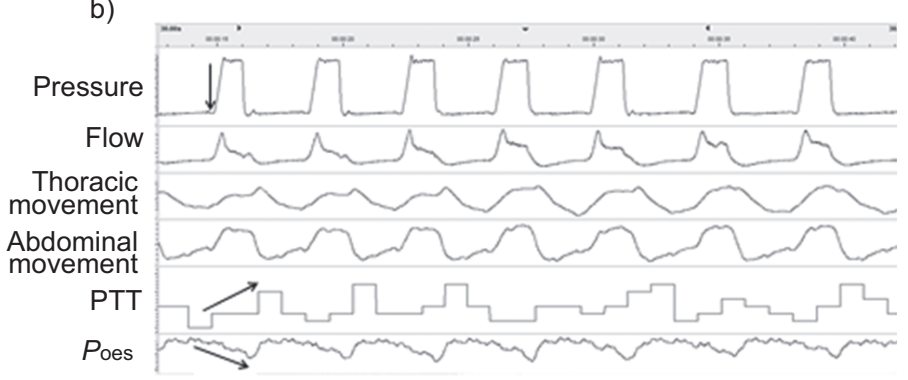

c)

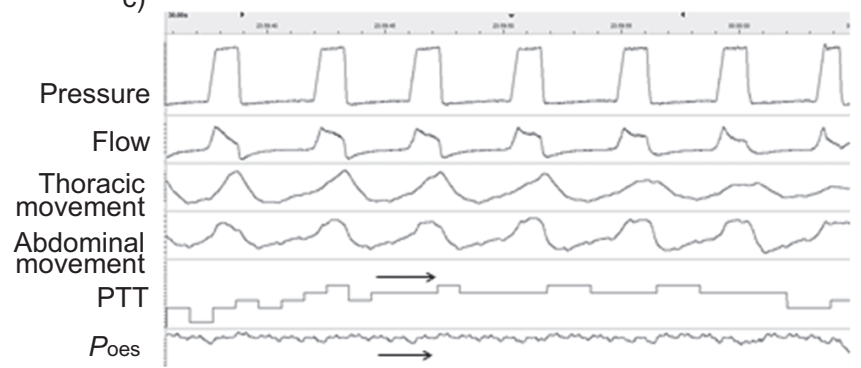

FIGURE 5. Ability of pulse transit time (PTT) to characterise respiratory effort during respiratory cycles triggered or not by the patients. a) Box 1: respiratory cycles are triggered by the patient; box 2: there is no inspiratory effort from the patient, all respiratory cycles are controlled by the ventilator. b) Respiratory cycles are triggered by the patient as demonstrated by the changes in pressure signal before pressurisation (arrow). There is a clear increase in respiratory effort as demonstrated by oesophageal pressure (Poes) and an increase in inspiratory-expiratory differences on PTT signal. c) All the respiratory cycles are controlled by the ventilator with an obvious reduction in effort both on PTT and Poes as compared with (b).

case in the presence of partial reduction of airflow: detecting whether persisting inspiratory efforts are increasing, suggesting upper airway obstruction, or not, suggesting a decrease in respiratory drive, requires an indicator of inspiratory effort, with Poes being the gold standard [4]. This is a key issue as both events lead to different strategies in terms of ventilator setting adjustment. Poes has been validated both in the intensive care unit for improving ventilators settings and in the field of sleep medicine as a marker of inspiratory effort [22]. However, as previously mentioned, routine use of Poes is questionable because of associated sleep disruption and leaks around the mask induced by the oesophageal probe. PTT has been suggested as a surrogate marker of inspiratory effort [23]. It has been documented as a reliable marker of inspiratory efforts in sleep studies performed in children with sleepdisordered breathing $[9,24,25]$ and in adults $[23,26]$. Our data support the use of PTT as a marker of inspiratory effort under bi-level positive pressure ventilation.
Using PTT for quantifying changes in inspiratory effort induced by NIV may also be of interest. It is usually admitted that efficacy of long-term NIV relies on resting the respiratory muscles, reducing the load against which the respiratory muscle pump must work and restoring central drive to breathing [27]. However, the tools currently available to assess muscle rest and load reduction all have significant limitations. As mentioned, oesophageal probes are poorly tolerated by patients and used only in specialised centres. Diaphragm or intercostal electromyograms are difficult to quantify, and require a level of expertise to be of practical interest. Data presented that PTT may provide a simple marker for assessing the degree of respiratory muscle rest provided when titrating pressure support under NIV. The changes in Poes and PTT were obvious during NIV without leaks, a situation associated with a complete rest of the respiratory muscles. In response to leaks, some normal subjects exhibited a higher percentage of triggered cycles and clearly increased their respiratory effort. 
This was nicely demonstrated by Poes but less clearly shown by PTT. This means that the PTT technique is easier to tolerate for the patients but probably less sensitive for detecting subtle increases in respiratory effort.

\section{Limitations of the study}

There are a certain number of limitations to the use of PTT. First, the quality of the signal is directly related to the quality of the ECG and pulse oximetry: these signals may easily become unstable, leading the analysis of PTT useless on bouts of polygraphy or PSG traces. Signal processing may also vary according to software and PSG or polygraphy devices used: this warrants further clinical testing. In addition, there is significant variation in PTT between individuals as a result of differences in blood pressure and vascular compliance. This does not unduly affect interpretation as it is the pattern of PTT change by which respiratory events are scored. From our own anecdotal experience, it is known that cardiac arrhythmias, such as atrial fibrillation, render the PTT signal almost uninterpretable. This is potentially a major drawback in some patients.

The sample size of our study was relatively limited but we performed systematic measurements of Poes during both diurnal and sleep experiments. Such an experimental design is difficult to implement in larger populations. At the end, we were able to draw significant conclusions from this well-defined population. Owing to a limited number of included subjects, we decided to investigate a homogeneous population of OHS patients. These patients appeared of particular interest as they exhibited both central and obstructive respiratory events during sleep. We anticipate that the ability of PTT to estimate respiratory effort will be the same in COPD and thoracic cage restrictive diseases. More questionable is the situation of patients with neuromuscular disorders producing only moderate respiratory effort. A specific study in this population is desirable.

PTT has the ability to quantitatively assess respiratory effort. However, during this study we did not try to separate hypopnoeas related to pharyngeal collapses from hypopnoeas owing to a decrease in ventilatory command associated with glottis closures.

PTT has the ability to quantitatively assess respiratory effort. However, during this study, we did not try to separate hypopnoeas related to pharyngeal collapse from hypopnoeas resulting from decrease in ventilatory command associated with glottic closure.

\section{Conclusion and perspectives}

Taking into account the aforementioned caveats, PTT is a promising tool for noninvasively assessing inspiratory muscle effort under bi-level positive pressure ventilation, and distinguishing between central and obstructive events under NIV not only in the sleep laboratory but also potentially in domiciliary sleep studies (polygraphy). The choice of software and its evolution is certainly critical to the development of PTT. Further studies are also required to assess PTT as a tool for identifying ineffective efforts under NIV and its capacity to be used for adjusting trigger sensitivity. The ability of PTT to estimate respiratory effort under NIV should be established in other patient populations, such as COPD and thoracic cage restrictive diseases.

\section{CLINICAL TRIAL}

This study is registered at www.clinicaltrials.gov with identifier NCT00983411.

\section{STATEMENT OF INTEREST}

None declared.

\section{REFERENCES}

1 Janssens JP, Borel JC, Pepin JL. Nocturnal monitoring of home non-invasive ventilation: the contribution of simple tools such as pulse oximetry, capnography, built-in ventilator software and autonomic markers of sleep fragmentation. Thorax 2010; 66: 438-445.

2 Berry RB, Chediak A, Brown LK, et al. Best clinical practices for the sleep center adjustment of noninvasive positive pressure ventilation (NPPV) in stable chronic alveolar hypoventilation syndromes. J Clin Sleep Med 2010; 6: 491-509.

3 Fanfulla F, Taurino AE, Lupo ND, et al. Effect of sleep on patient/ ventilator asynchrony in patients undergoing chronic non-invasive mechanical ventilation. Respir Med 2007; 101: 1702-1707.

4 Gonzalez-Bermejo J, Perrin C, Janssens JP, et al. Proposal for a systematic analysis of polygraphy or polysomnography for identifying and scoring abnormal events occurring during noninvasive ventilation. Thorax 2012; 67: 546-552.

5 Guo YF, Sforza E, Janssens JP. Respiratory patterns during sleep in obesity-hypoventilation patients treated with nocturnal pressure support: a preliminary report. Chest 2007; 131: 1090-1099.

6 Pitson DJ, Stradling JR. Value of beat-to-beat blood pressure changes, detected by pulse transit time, in the management of the obstructive sleep apnoea/hypopnoea syndrome. Eur Respir J 1998; 12: 685-692.

7 Argod J, Pepin JL, Levy P. Differentiating obstructive and central sleep respiratory events through pulse transit time. Am J Respir Crit Care Med 1998; 158: 1778-1783.

8 Argod J, Pepin JL, Smith RP, et al. Comparison of esophageal pressure with pulse transit time as a measure of respiratory effort for scoring obstructive nonapneic respiratory events. Am J Respir Crit Care Med 2000; 162: 87-93.

9 Pepin JL, Delavie N, Pin I, et al. Pulse transit time improves detection of sleep respiratory events and microarousals in children. Chest 2005; 127: 722-730.

10 Wilson TA, Angelillo M, Legrand A, et al. Muscle kinematics for minimal work of breathing. J Appl Physiol 1999; 87: 554-560.

11 Contal O, Vignaux L, Combescure C, et al. Monitoring of noninvasive ventilation by built-in software of home bi-level ventilators: a bench study. Chest 2012; 141: 469-476.

12 Bachy JP, Eberhard A, Baconnier P, et al. A program for cycleby-cycle shape analysis of biological rhythms. Application to respiratory rhythm. Comput Methods Programs Biomed 1986; 23: 297-307.

13 Pitson DJ, Sandell A, van den Hout R, et al. Use of pulse transit time as a measure of inspiratory effort in patients with obstructive sleep apnoea. Eur Respir J 1995; 8: 1669-1674.

14 Mokhlesi B. Obesity hypoventilation syndrome: a state-of-the-art review. Respir Care 2010; 55: 1347-1362.

15 Piper AJ, Grunstein RR. Obesity hypoventilation syndrome: mechanisms and management. Am J Respir Crit Care Med 2011; 183: 292-298.

16 Borel JC, Tamisier R, Gonzalez-Bermejo J, et al. Noninvasive ventilation in mild obesity hypoventilation syndrome: a randomized controlled trial. Chest 2012; 141: 692-702.

17 Chouri-Pontarollo N, Borel JC, Tamisier R, et al. Impaired objective daytime vigilance in obesity-hypoventilation syndrome: impact of noninvasive ventilation. Chest 2007; 131: 148-155. 
18 Bland JM, Altman DG. Statistical methods for assessing agreement between two methods of clinical measurement. Lancet 1986; 1: 307-310.

19 McGraw KO, Wong SP. Forming inferences about some intraclass correlation coefficients. Psychol Methods 1996; 1: 30-46.

20 Rabec C, Rodenstein D, Leger P, et al. Ventilator modes and settings during non-invasive ventilation: effects on respiratory events and implications for their identification. Thorax 2010; 66: 170-178.

21 Janssens JP, Borel JC, Pepin JL. Nocturnal monitoring of home noninvasive ventilation: the contribution of simple tools such as pulse oximetry, capnography, built-in ventilator software and autonomic markers of sleep fragmentation. Thorax 2010; 66: 438-445.

22 Fanfulla F, Delmastro M, Berardinelli A, et al. Effects of different ventilator settings on sleep and inspiratory effort in patients with neuromuscular disease. Am J Respir Crit Care Med 2005; 172: 619-624.
23 Pepin JL, Tamisier R, Borel JC, et al. A critical review of peripheral arterial tone and pulse transit time as indirect diagnostic methods for detecting sleep disordered breathing and characterizing sleep structure. Curr Opin Pulm Med 2009; 15: 550-558.

24 Brietzke SE, Katz ES, Roberson DW. Pulse transit time as a screening test for pediatric sleep-related breathing disorders. Arch Otolaryngol Head Neck Surg 2007; 133: 980-984.

25 Huang H, Ye JY, Li YR, et al. Pulse transit time for quantifying inspiratory effort in patients with obstructive sleep apnea. ORL J Otorhinolaryngol Relat Spec 2011; 73: 53-60.

26 Poyares D, Guilleminault C, Rosa A, et al. Arousal, EEG spectral power and pulse transit time in UARS and mild OSAS subjects. Clin Neurophysiol 2002; 113: 1598-1606.

27 Elliott MW. Domiciliary non-invasive ventilation in stable COPD? Thorax 2009; 64: 553-556. 